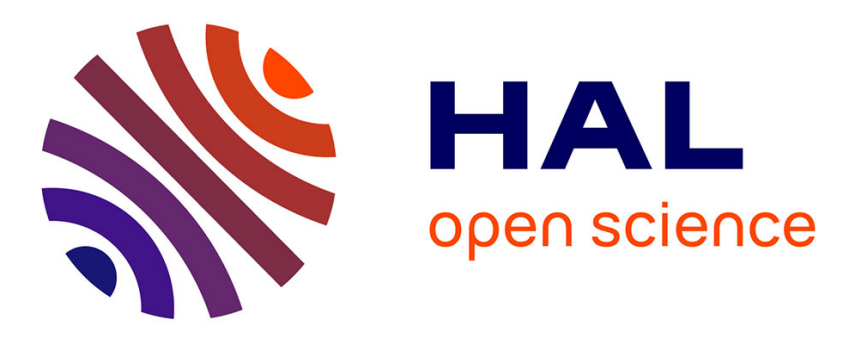

\title{
An image-guided atomistic reconstruction of pyrolytic carbons
}

\author{
Jean-Marc Leyssale, Jean-Pierre da Costa, Christian Germain, Patrick \\ Weisbecker, Gerard L. Vignoles
}

\section{- To cite this version:}

Jean-Marc Leyssale, Jean-Pierre da Costa, Christian Germain, Patrick Weisbecker, Gerard L. Vignoles. An image-guided atomistic reconstruction of pyrolytic carbons. Applied Physics Letters, 2009, 95 (23), pp.231912-1. 10.1063/1.3272949 . hal-00484786

\section{HAL Id: hal-00484786 https://hal.science/hal-00484786}

Submitted on 19 May 2010

HAL is a multi-disciplinary open access archive for the deposit and dissemination of scientific research documents, whether they are published or not. The documents may come from teaching and research institutions in France or abroad, or from public or private research centers.
L'archive ouverte pluridisciplinaire HAL, est destinée au dépôt et à la diffusion de documents scientifiques de niveau recherche, publiés ou non, émanant des établissements d'enseignement et de recherche français ou étrangers, des laboratoires publics ou privés. 


\title{
An image-guided atomistic reconstruction of pyrolitic carbons
}

\author{
Jean-Marc Leyssale, ${ }^{1}$ Jean-Pierre Da Costa, ${ }^{2}$ Christian Germain, ${ }^{2}$ Patrick Weisbecker, ${ }^{2}$ \\ and Gérard L. Vignoles ${ }^{2}$ \\ 1) LCTS, UMR 5801 CNRS-CEA-Snecma Propulsion Solide-Université Bordeaux 1, \\ 33600 Pessac, France \\ 2) IMS, UMR 5218 Université Bordeaux 1/ENSEIRB/ENSCPB/ENITAB, \\ 33405 Talence, France
}

(Dated: 28 October 2009)

A new method for the generation of atomistic models of dense nanotextured carbons is presented. This method is based on the statistical analysis of HRTEM images and their three-dimensional extension through image synthesis under constraint. The resulting 3D images then serve as an external potential bringing the atoms to settle preferentially on the black areas during a conventional simulated annealing simulation. Application of this method to the case of two laminar pyrocarbons, differing in their degree of disorder, highlights the promising nature of this approach. 
Atomistic reconstruction methods are nowadays well-established tools for linking experimental characterization data to the atomic scale structure of matter ${ }^{1}$. Most of them are based on the reproduction of orientation-averaged structural features like the pair distribution functions (PDFs) so that these methods are usually very efficient for isotropic systems. However, when dealing with materials displaying a neatly anisotropic nanotexture, like most dense graphene-based carbons, the success of such approaches relies strongly on the initial guess of the atomic structure ${ }^{2}$. For instance, two PDF-based computer reconstructions of the same material ${ }^{3}$ show a drastically different nanotexture: one, quite isotropic, is a disordered carbon with a dominating $s p^{2}$ character $^{4}$ while the other one is a strongly anisotropic stack of faulted graphene sheets ${ }^{5}$.

In most scientific papers, the nanotexture of turbostratic carbons is described from High Resolution Transmission Electron Microscopy (HRTEM) lattice fringe images ${ }^{6}$ rather than in terms of PDF analysis. For instance, we show in Fig. 1 the HRTEM images of a rough laminar pyrocarbon (pyC), used as a matrix in many $\mathrm{C} / \mathrm{C}$ composites $^{7}$, as prepared (AP) and after heat treatment (HT). These images actually carry most of the information on the extension and stacking properties of graphene sheets in the materials: the HT pyC shows more extended and flatter fringes than the AP one. Although the information provided by this technique is mainly qualitative, recent developments in image analysis methods allow a finer description of the nanotexture to be $\operatorname{drawn}^{8-10}$. In this letter we propose a new atomistic reconstruction strategy based on (i) the statistical analysis of HRTEM images, (ii) the synthesis of 3D HRTEM-like images and (iii) a simulated annealing simulation guided by the former 3D images. This method is applied to the two materials of Fig. 1.

To synthesize 3D HRTEM-like images from experimental (2D) images, we use an original pyramidal analysis/synthesis scheme derived from a standard 2D approach ${ }^{11}$. This novel procedure, which will be detailed in a forthcoming publication ${ }^{12}$, can be summarized as follows:

First, the HRTEM image sample is decomposed into a set of multi-resolution subbands using the steerable pyramid decomposition ${ }^{11}$ and a statistical analysis of the subbands is performed to produce of a pyramidal collection of first order (mean, variance, skewness, kurtosis) and second order (autocorrelation coefficients) 2D statistics. Such an analysis is performed on experimental images after application of a band-pass filter to remove lowfrequency (background gradients) and high-frequency (noise) artifacts. Filtering also makes 

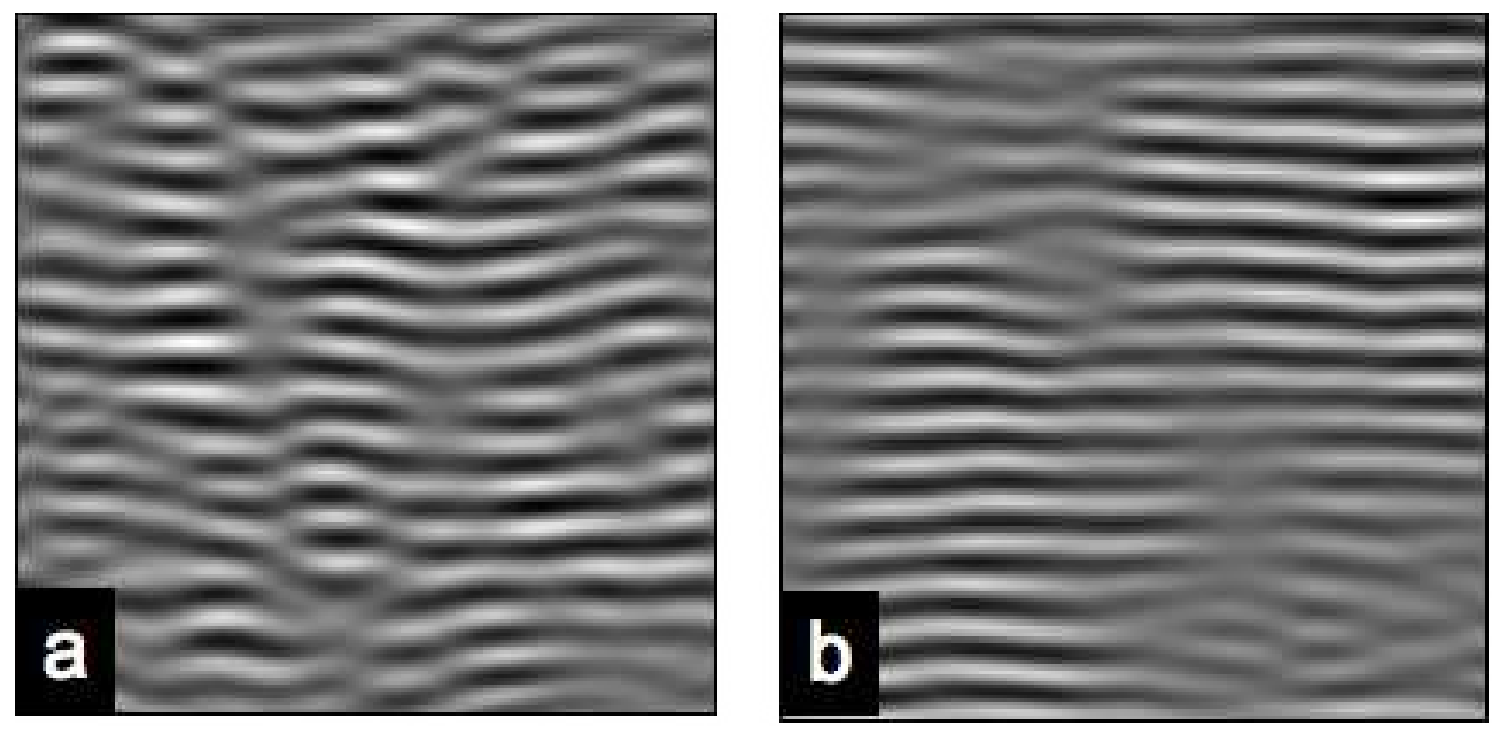

FIG. 1. HRTEM lattice finge images of AP (a) and HT (b) pyCs (low and high frequency artifacts have been filtered).

the images periodic, a suitable property for image synthesis and atomistic simulations. In a second step, the collected 2D statistics are extended to a pyramidal collection of 3D target statistics. This is trivial for first order statistics. The main difficulty is in determining target 3D autocorrelation coefficients from the reference 2D autocorrelation coefficients. Such statistics describe the dependence relationship between two pixels in a texture (respectively two voxels in a solid texture). In the case of pyrocarbons (and more generally turbostratic carbons), we use the property of orthotropy, namely that all directions perpendicular to the main stacking direction (vertical on Fig. 1) have the same statistical properties, to obtain the target 3D autocorrelation coefficients. Finally, an initially random 3D image block is iteratively decomposed using a 3D pyramidal decomposition, modified to meet the 3D target statistics and reconstructed, until it satisfies the multi-resolution 3D target statistics.

The 3D images synthesized from the HRTEM images of Fig. 1 are shown in Fig. 2. They have been obtained using a three levels and four orientations pyramidal decomposition and 7x7 neighborhoods autocorrelation coefficients. It is obvious to see the correspondance between the textures of Figs 1 and 2. In both cases, images of the AP material (Figs 1a and 2a) show short and wavy fringes while those of the HT material (Figs 1b and 2b) exhibit long and flat fringes.

Adopting typical pyrocarbon values of the interlayer spacing $d_{002}$ and of the density, 

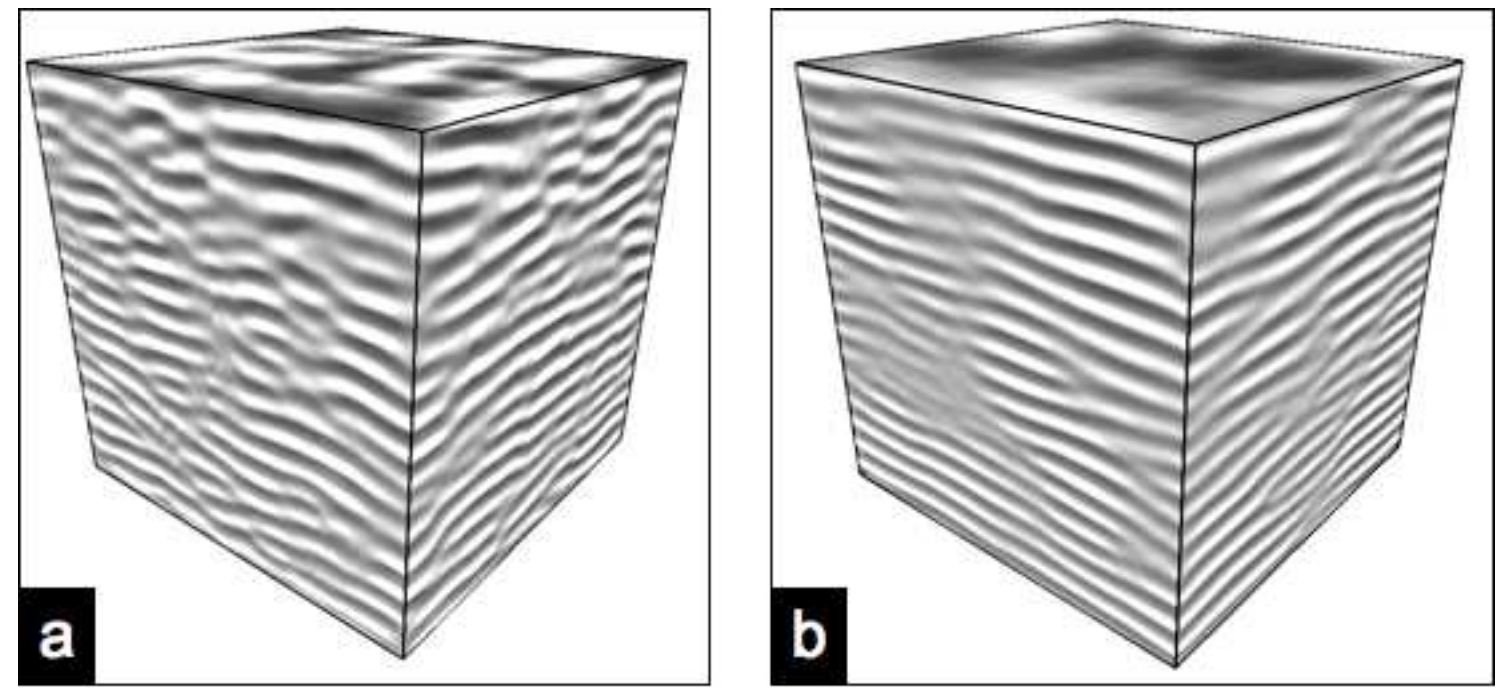

FIG. 2. 3D HRTEM-like images of AP (a) and HT (b) pyCs.

namelely $3.5 \AA$ And $2.1 \mathrm{~g} / \mathrm{cm}^{3}$ and considering that the 3D images counts on average 16 (AP) and 17 (HT) fringes, simulation boxes of the suited sizes are randomly (with the condition that no C-C distance falls below $1.3 \AA$ ) filled with respectively 18507 and 22198 carbon atoms (no heteroatoms are considered in this work). Random initial velocities corresponding to a high temperature $(8000 \mathrm{~K})$ are then assigned to the systems and two interaction potentials $V_{R E B O}$ and $V_{H R T E M}$ are switched on. The first one, $V_{R E B O}$ is a reactive interatomic potential which properly renders the different bonding possibilities of carbon as well as the formation and breaking of $\mathrm{C}-\mathrm{C}$ bonds ${ }^{13}$. This potential thus ensures the atomic models to possess chemically realistic structures. The second one, $V_{H R T E M}$, aims at bringing the atoms on the fringes (the black regions on Figs $2 \mathrm{a}$ and $2 \mathrm{~b}$ ). We chose here $V_{H R T E M}=k_{I m} I\left(\mathbf{r}_{i}\right)$, a simple linear function where $k_{I m}$ is a proportionality factor and $I\left(\mathbf{r}_{i}\right)$ is the greyscale level (ranging from 0 to 1 for respectively black and white voxels) at the location $\mathbf{r}_{i}$ of atom $i$. A simulated annealing procedure is then launched using molecular dynamics together with a stochastic thermostat ${ }^{14}$. During this simulation the system is "slowly" cooled from its initial temperature down to $0 \mathrm{~K}$ by adjusting the target temperature of the thermostat at each timestep. Such a procedure mimics the dynamics of a carbon system cooled from the liquid state in the presence of an external field (the 3D image potential) bringing the system to a given nanotexture.

Preliminary simulations performed on 13 fringes height images (not presented here) showed that a value of $k_{I m}=2 e V\left(\approx 1 / 4^{t h}\right.$ of a carbon atom energy) gives a good bal- 
ance between finding low energy structures and obtaining the suited nanotexture. These simulations also revealed that the following cooling squence (fast at high and low temperatures and slow close to the carbon melting point) is an optimal solution: $125 \mathrm{~K} / \mathrm{ps:}$ [8000, $7000 \mathrm{~K}] \&[2000,0 \mathrm{~K}], 25 \mathrm{~K} / \mathrm{ps}:[7000,6000 \mathrm{~K}] \&[3000,2000 \mathrm{~K}], 5 \mathrm{~K} / \mathrm{ps}:[6000,5000 \mathrm{~K}] \&$ [4000, $3000 \mathrm{~K}]$ and $1 \mathrm{~K} / \mathrm{ps}$ : [5000, $4000 \mathrm{~K}]$. This temperature ramp gives lower final energies than for instance a much costly uniform cooling at $2 \mathrm{~K} / \mathrm{ps}$. After completion of the annealing, $V_{H R T E M}$ is switched off and the system is relaxed at $300 \mathrm{~K}$ for some picoseconds using the AIREBO potential ${ }^{15}$ (a modification of REBO, incorporating van der Waals interactions between non-bonded atoms).
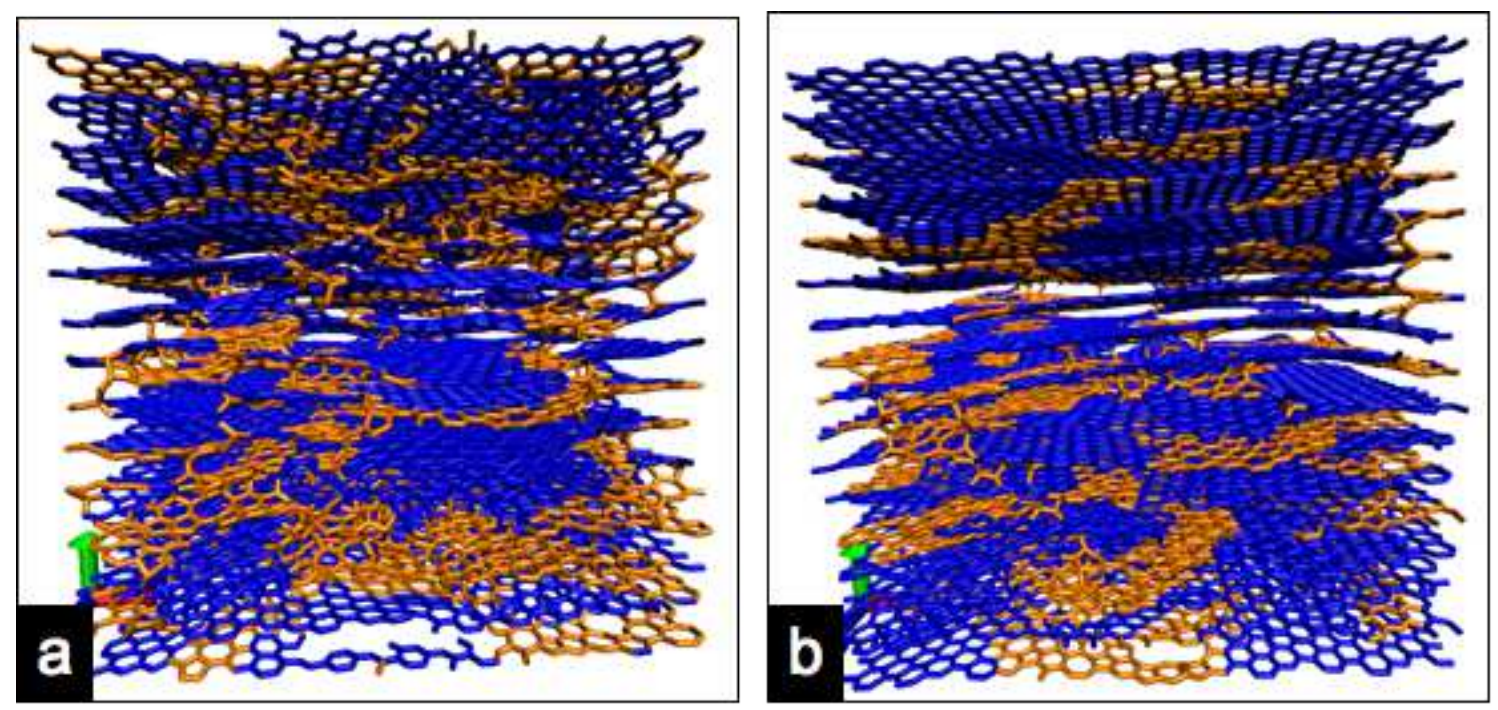

FIG. 3. Reconstructed atomistic models of AP (a) and HT (b) pyCs (blue: pure $C_{6}$ rings; orange: other C-C bonds).

The atomistic configurations obtained for the two materials are shown in Fig. 3. The two models show a well pronounced laminar nanotexture, typical of pyrocarbons. Carbon bonding is almost entirely of $s p^{2}$ nature for both models (97 and $99 \%$ for respectively AP and HT pyCs), the remaining carbon atoms being mainly $s p^{3}$ hybridized. In order to better characterize the materials, we performed an analysis of "shortest path ring" (SPR $)^{16}$ statistics, limited to three-coordinated atoms. A similar repartition of rings is found in the two materials with respectively 88.6 and $88.9 \%$ of six-member rings $\left(C_{6}\right), 5.5$ and $5.4 \%$ of 5-member rings $\left(C_{5}\right)$ and 5.7 and $5.5 \%$ of 7 -member rings $\left(C_{7}\right)$ for respectively the $\mathrm{AP}$ and HT models. In Fig. 3, pure $C_{6}$ rings, namely those $C_{6}$ rings whose atoms belong only 
to $C_{6}$ rings, are shown with blue sticks while other $\mathrm{C}-\mathrm{C}$ bonds are shown in orange. Visual comparison of Figs $3 \mathrm{a}$ and $3 \mathrm{~b}$ shows that pure $C_{6}$ domains appear more extended and flat in HT than in AP. Even if the fractions of non hexagonal rings are similar, they are distributed differently in the two materials. Particularly, while apparently scattered in the AP pyC, thus creating curvatures, an important fraction of non hexagonal rings segregates in rather flat domains (Fig.3b) in the FT model. These domains with many neighboring $C_{5}$ and $C_{7}$ rings are compatible with flat textures (e. g. Stone-Wales defects) and are prone to form hexagonal rings under further annealing or graphitization.
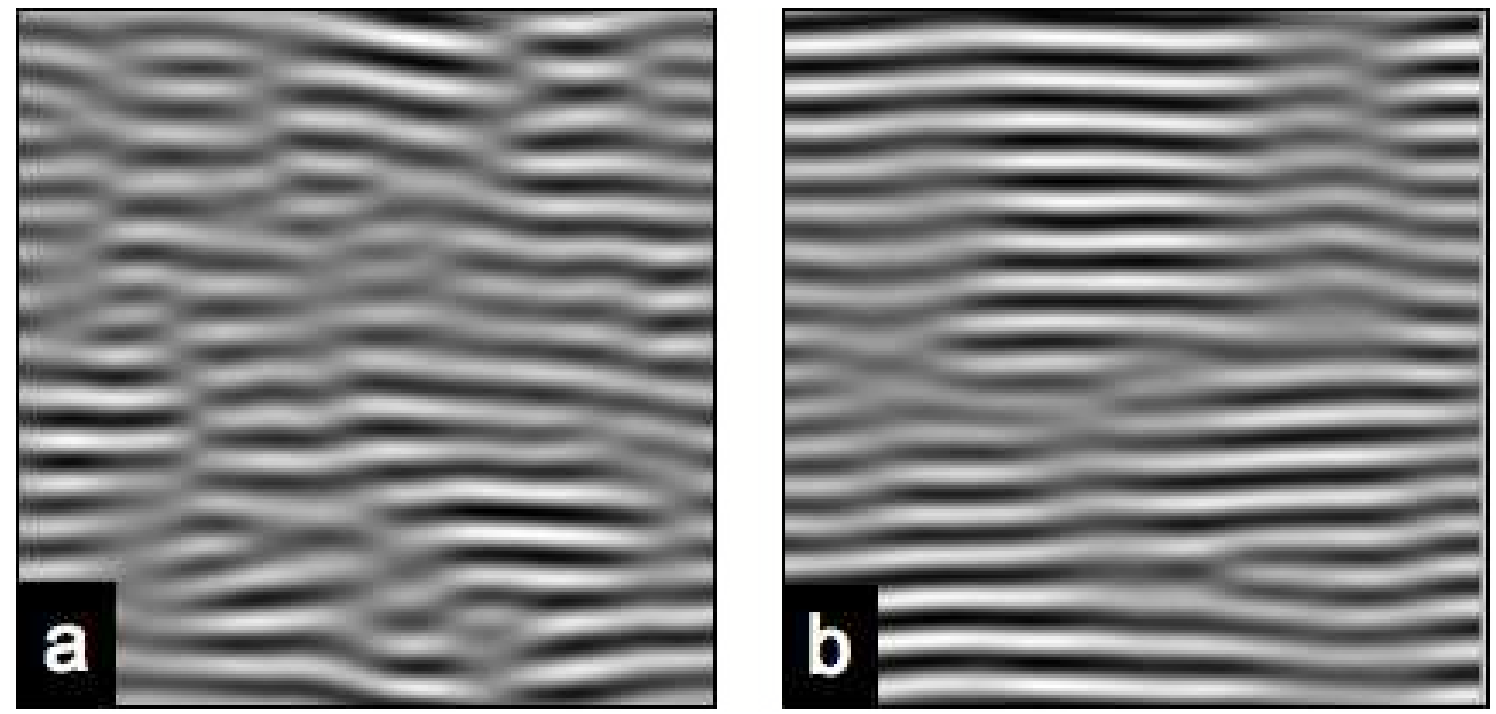

FIG. 4. Simulated HRTEM images obtained from the reconstructed atomistic models of the AP (a) and HT (b) pyCs.

Going back to the very beginning of this work, we show in Fig. 4 the HRTEM images simulated from the two atomistic models using the NCEMSS software ${ }^{17}$. The similarity in terms of fringes lengths and undulations between initial images (Figs 1a and 1b) and their corresponding simulated images (Figs $4 \mathrm{a}$ and $4 \mathrm{~b}$ ) is obvious and confirm that the atomistic models contain most, if not all, of the nanotextural information present in the experimental images.

Finally, we plot on Fig. 5 the Reduced Pair Distribution Functions (RPDFs) ${ }^{18}$ computed for the AP model, the HT model and an ideal graphite lattice as well as the experimental RPDF of a nanoporous carbon ${ }^{3}$ (unfortunately, we are not aware of any experimental RPDF of a pyC). Two conlusions come immediately when looking at Fig. 5: (i) even though the 


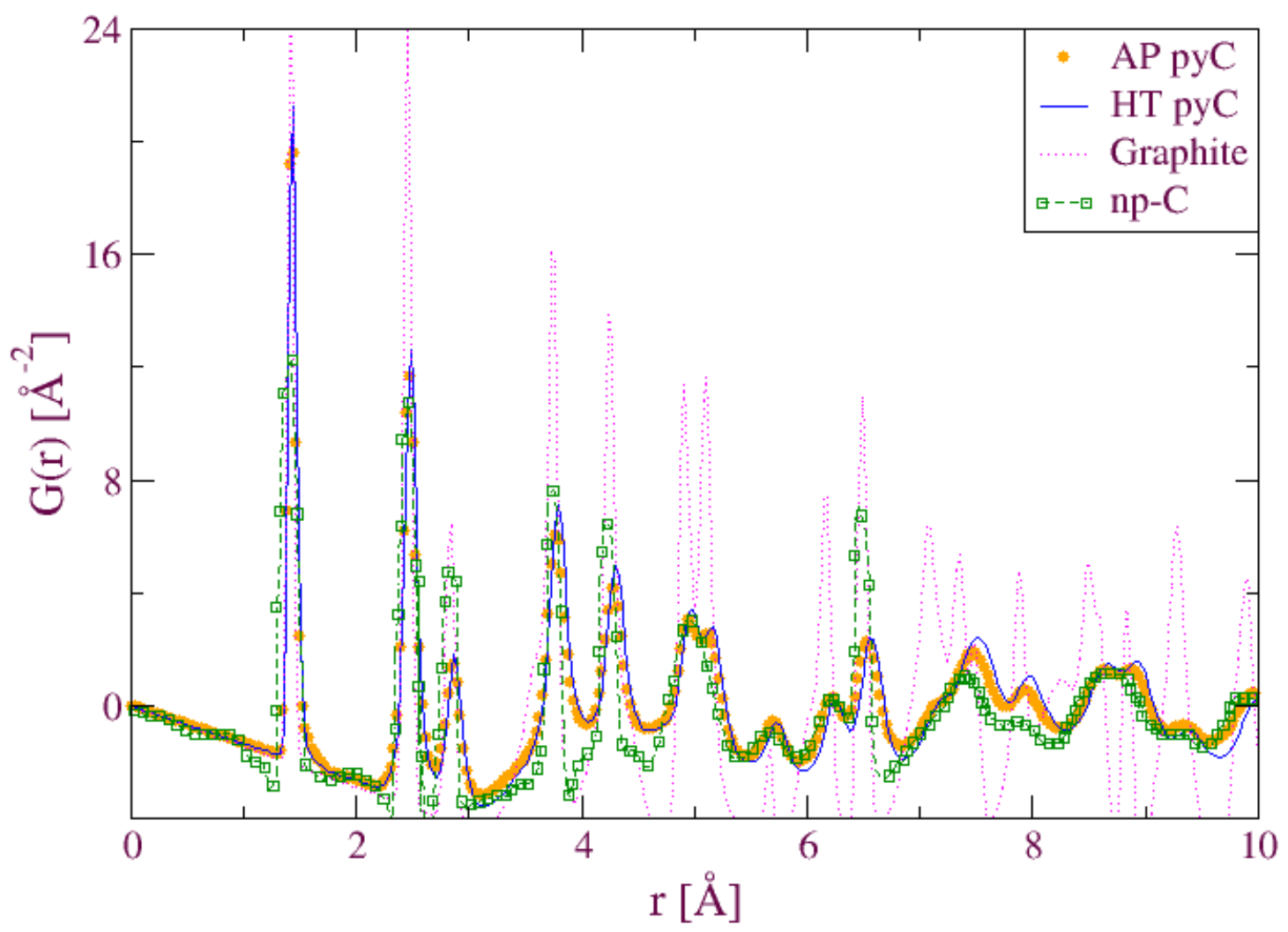

FIG. 5. RPDFs of the atomistic models of AP (full circles) and HT (straight line) pyCs. Also displayed are an ideal hexagonal graphite [computed] (dotted) and a nanoporous carbon ${ }^{3}$ [experimental](dashed line and empty squares).

two pyCs present rather different nanotextures as shown on the HRTEM images and/or atomistic configurations, their RPDFs are almost identical (the HT model showing only very slightly higher peaks than the AP one). This shows how very little information on the nanotexture is carried by the RDPF; (ii) Although they comprise all the peaks typical of turbostratic carbons (compare to graphite and to the carbon of Petkov ${ }^{3}$, the RPDFs of both pyC models show rather broad peaks, indicating a probably too high degree of nonhexagonal rings (Petkov's PDF has been modeled with atomistic models comprising zero ${ }^{4}$ to $5 \%^{5}$ of such rings).

To summarize, the method presented here, based on HRTEM images analysis and synthesis as well as atomistic simulations allowed us to build atomistic models of pyrocarbons whose nanotextures are in excellent agreement with the real materials. However, both the ring anlysis and the observation of the RPDFs seem to indicate that our models counts 
too many non-hexagonal rings $(11 \%)$. Nevertheless, we have reasons to believe that this "chemical quality" can be neatly improved by lowering the rate of the simulated annealing process or by including in this process, together with the 3D image, a penalty term based on an experimental RPDF, when available. Such an approach has been used by Jain et al. ${ }^{19}$ in the simpler case of low density porous carbons. Furthermore, introduction of $\mathrm{H}$ atoms in the simulated structures will also help in this direction.

\section{ACKNOWLEDGMENTS}

We thank R. Vitti for his contribution to the early stage of this project as well as Dr R. J.-M. Pellenq for useful discussions and advices.

\section{REFERENCES}

${ }^{1}$ M. T. Dove, M. G. Tucker, S. A. Wells, and D. A. Keen, EMU Notes in Mineralogy 4, 59 (2002).

${ }^{2}$ P. Zetterström, S. Urbonaite, F. Lindberg, R. G. Delaplane, J. Leis, and G. Svensson, J. Phys.: Condens. Matter 17, 3509 (2005).

${ }^{3}$ V. Petkov, R. G. Di Francesco, S. J. L. Billinge, M. Acharya, and H. C. Foley, Phil. Mag. B 79, 1519 (1999).

${ }^{4}$ M. Acharya, M. Strano, J. P. Mathews, S. J. L. Billinge, V. Petkov, S. Subramoney, and

H. C. Foley, Phil. Mag. B 79, 1499 (1999).

${ }^{5}$ M. A. Smith, H. C. Foley, and R. F. Lobo, Carbon 42, 2041 (2004).

${ }^{6}$ A. Oberlin, Carbon 40, 7 (2002).

${ }^{7}$ G. L. Vignoles, F. Langlais, C. Descamps, A. Mouchon, H. Le Poche, N. Bertrand, and

N. Reuge, Surf. Coat. Tech. 188-189, 241 (2004).

${ }^{8}$ H. S. Shim, R. H. Hurt, and N. Y. C. Yang, Carbon 38, 29 (2000).

${ }^{9}$ J.-N. Rouzaud and C. Clinard, Fuel Process. Tech. 77-78, 229 (2002).

${ }^{10}$ C. Germain, J.-P. Da Costa, O. Lavialle, and P. Baylou, Signal Process. 83, 229 (2003).

${ }^{11}$ J. Portilla and E. P. Simoncelli, Int. J. Comput. Vis. 40, 49 (2000).

${ }^{12}$ J.-P. Da Costa and C. Germain, to-be -published. 
${ }^{13}$ D. W. Brenner, O. A. Shenderova, J. A. Harrison, S. J. Stuart, B. Ni, and S. B. Sinnott, J. Phys. Condens. Matter. 14, 783 (2002).

${ }^{14}$ M. P. Allen and D. J. Tildesley, Computer Simulation of Liquids (Oxford University Press, 1987).

${ }^{15}$ S. J. Stuart, A. B. Tutein, and J. A. Harrison, J. Chem. Phys. 112, 6472 (2000).

${ }^{16}$ D. S. Franzblau, Phys. Rev. B 44, 4925 (1991).

${ }^{17}$ R. Kilaas, in Proceedings of 22nd Annual Conference of the Microbeam Analysis Society (1987).

${ }^{18}$ T. Egami and S. J. L. Billinge, Underneath the Bragg Peaks (Pergamon, 2003).

${ }^{19}$ S. K. Jain, R. J.-M. Pellenq, J. P. Pikunic, and K. E. Gubbins, Langmuir 22, 9942 (2006). 